\title{
A new species of Sauromatum (Araceae) from North-East India
}

\author{
Umeshkumar L. Tiwari*, Rohan Maity and Sudhansu Sekhar Dash¹ \\ Botanical Survey of India, Arunachal Pradesh Regional Centre, Senki View, Itanagar-791111, India \\ ${ }^{1}$ Botanical Survey of India, CGO Complex, 3rd MSO Building, Block F, 5th Floor, DF Block, Sector I \\ Salt Lake City, Kolkata-700064 \\ *Corresponding author: ukltiwari.bsi@nic.in, tigerumesh11@gmail.com

\section{पूर्वोत्तर भारत से सौरोमेटम (एरेसी) की एक नवीन जाति का अन्वेषण}

उमेशकुमार एल. तिवारी, रोहन मैती एवं सुधांशु शेखर दास

\section{सारांश}

भारत के अरुणाचल प्रदेश से सौरोमेटम (एरेसी - ऐरेई) की एक नवीन जाति को अन्वेषित एवं वर्णित किया गया है। इसकी सही एवं सरल पहचान हेतु विस्तृत वर्णन, डिजिटल छायाचित्र, निकट समवर्ती जातियों से इसके आकारिकीय लक्षणों की तुलना एवं वैश्विक स्तर पर पाये जाने वाली जातियों पर आधारित कृत्रिम वर्गिकी कुंजी भी दी गई है।

\section{ABSTRACT}

A new species of Sauromatum (Araceae: Areae) is described here from Arunachal Pradesh, India. Detailed description, digital photographs, comparison of morphological characters with closely allied species and artificial key for globally found species is provided for easy identification.

Keywords: Areae, Arunachal Pradesh, Eastern Himalaya, sp. nov.

\section{INTRODUCTION}

The genus Sauromatum Schott (1832: 17) of family Araceae comprises of c. 10 species (POWO, 2021) distributed in Tropical Africa, Tropical \& Subtropical South-East Asia-Bangladesh, Bhutan, China, Combodia, India, Indonesia, Myanmar, Nepal, Thailand and Vietnam (Hooker 1893; Hetterscheild \& Boyce 2000; Hetterscheild \& al., 2001; Heng \& Hetterscheild 2010; Cusimano \& al., 2010; Boyce \& al., 2012). Since the publication of Flora of British India (Hook.f., 1893), no significant additions have been made on the genus Sauromatum from Indian perspective till last decade. During the past one decade one new species Sauromatum meghalayense D.K. Roy
\& al. has been described from Meghalaya, India (Talukdar \& al., 2014) and one new distributional record (Sauromatum horsfieldii Miq. (1856: 196) is from Nagaland (Odyuo \& al., 2015) has been made for Indian flora. Sauromatum diversifolium (Wall. ex Schott) Cusimano \& Hett. (2010: 445) and Sauromatum venosum (Dryand. ex Aiton) Kunth (1841: 281) are reported from Arunachal Pradesh (Nangkar \& Tag 2018, 2019; Roy 2018) as new addition for the state flora. Sasikala \& al. (2019) reported 50\% worldwide representative of the genus Sauromatum are occurring in India.

During our recent field exploration in Papum Pare district of Arunachal Pradesh, India, an interesting plant of the genus Sauromatum Schott (1832) was collected. 


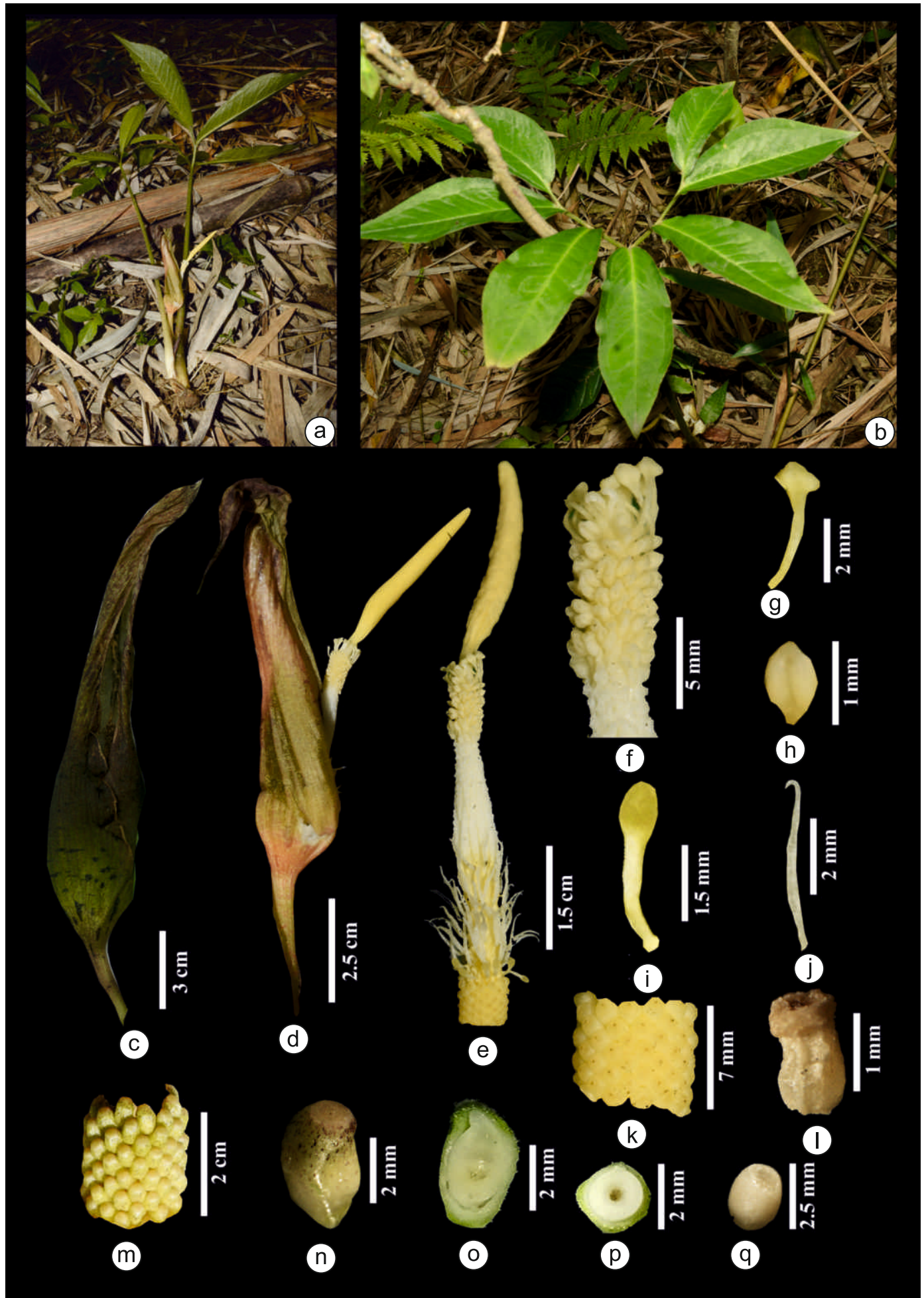

Fig. 1. Sauromatum arunachalense U.L. Tiwari, R. Maity \& S.S. Dash. a. Habit and Habitat; b. Leaf; c. Spathe front view, d. Spathe side view; e. Spadix; f. Staminate flowers zone; g. Upper staminate flower; h. Lower staminate flower; i. Basal staminode; j. Sub-basal staminode; k. Pistillate flowers zone; 1. Pistillate flower; m. Infructescence; $n$. Fruit; O. L.S. of fruit; P. T.S. of fruit; Q. Seed. 
A comprehensive review of literature on the genus and careful study of specimens in flowering and fruiting stages were done, still the identity of the entity could not be fixed. All the important morphological characters of the leaf blade, petiole, inflorescence, spathe tube, cylindrical appendix, fruiting zone of spadix, berries, etc., are found to be distinct from all the known species of the genus. Therefore, the plant is described here as Sauromatum arunachalense sp. nov. hitherto undescribed.

A photo-plate showing habit and all morphological characters, an artificial key to the species and a comparative table (Table 1) based on morphological characters with its closely allied species are also provided here for easy identification.

\section{TAXONOMIC TREATMENT}

Sauromatum arunachalense U.L. Tiwari, R. Maity \& S.S. Dash, sp. nov. (Fig. 1)

Type:- INDIA, ARUNACHAL PRADESH: Papum Pare District, Senki View, 27.096716 ${ }^{\circ} \mathrm{N}, 93.605687^{\circ} \mathrm{E}$, 272 m, 28 March 2020, U.L. Tiwari, 41100 (holotype: ARUN!)

Diagnosis:- Sauromatum arunachalense U.L. Tiwari, R. Maity \& S.S. Dash closely allied to S. horsfieldii Miq. but distinguished by presence of pedatisect leaf with 57 oblanceolate leaflets (vs 7-12, elliptic-lanceolate leaflets), staminate flowers of 2 types with stalk of different size (vs all staminate flowers with minute stalk of equal length), sub-basal neuter flowers with hooked apex (vs sub-basal neuter flowers only bent upward), ovary longitudinally ridged on outer surface (vs ovary smooth on outer surface) and fruits 1-seeded (vs fruits 2-seeded).

Tuberous herbs, $20-30 \mathrm{~cm}$ high. Tuber depressed globose, $c$. $1 \mathrm{~cm}$ high, $1.5-2.0 \mathrm{~cm}$ in diam., light brownish green outside, white inside. Leaf pedatisect; leaflets 5-7, sessile, oblanceolate, base oblique, margin entire to distantly crenate-undulate, apex caudate acuminate, glabrous; mature middle leaflet 10-15 $\times$ 2-4 cm, usually equal or smaller than adjacent lateral ones; lateral leaflets $4-15 \times 1-4 \mathrm{~cm}$, gradually smaller; petiole terete, $18-20(-30) \mathrm{cm}$ long, $4-6 \mathrm{~mm}$ in diam., glabrous, green, sometimes basal portion with purplish tinge. Inflorescence single, appearing in conjunction with leaf; peduncle terete, longitudinally ridged when dried, $2.5-6.0 \mathrm{~cm}$ long, $2-5 \mathrm{~mm}$ in diam., glabrous, green. Spathe lanceolate, 5.5-11(-12) cm long, convolute at base, apex long caudate, glabrous on both surfaces, longitudinally ridged outside when mature, pinkish straw-light green with pinkish longitudinal stripes and purple spots throughout, dark purple at maturity, inside smooth, spongy-soft, greenish-white towards base. Spadix shorter than spathe, $5-6(-9) \mathrm{cm}$; female zone cylindric, 5-7 $\times 3-5 \mathrm{~mm}$; pistillate flowers densely arranged; ovary oblong, longitudinally ridged, uniloculate, white; stigma sessile, ring like, surrounding depression; sterile zone narrowly conical, c. $2 \mathrm{~cm}$ long; staminodes of two types; proximal staminodes few, spatulate, white, brown when dry, 2-4 mm long; subbasal staminodes subulate, strongly bent upwards, apex curved, hook like, white, suddenly shorter towards upper portion; interstice naked, grooved, twice longer than sterile zone, gradually narrower towards apex; male zone cylindrical, $0.8-1.5 \mathrm{~cm}$ long, gradually wider towards apex, 3-4 $\mathrm{mm}$ wide at apex; male flowers of two types, cream-white, $0.2-2.0 \mathrm{~mm}$ long, with dissimilar stalks, upper flowers with c. $1 \mathrm{~mm}$ long stalk, basal flowers with minute stalk; appendix clavate, shortly stipitate, smooth, erect, 30-40 $\times 4-6 \mathrm{~mm}$, cream yellow, rusty brown when dried, apex rounded; infructescence cylindric, 20-30 mm long; fruits semicylindric, 3-4 mm long, gradually wider towards apex, apex truncate, whitish green towards base, gradually greener towards apex, purple spots from apex to middle, 1 seeded. Seeds oblong-oval, 2-3 mm long; seed coat with reticulate ornamentation.

Habitat and distribution:- Found growing under shade of Bambusa pallida, Castanopsis indica, Duabanga grandiflora, Dendrocalamus hamiltonii etc, between elevation of 270-275 m; currently known only from the type locality Senki View, Papum Pare district of Arunachal Pradesh, India.

Flowering: March-April. Fruiting: April-May.

Etymology:- The specific epithet of this new species refers to the state Arunachal Pradesh from where it is discovered.

Additional specimen examined: INDIA, Arunachal Pradesh: Papum Pare District, Senki View, $27.0966876^{\circ} \mathrm{N}, 93.6055112^{\circ} \mathrm{E}, 270 \mathrm{~m}, 11$ April 2021, U.L. Tiwari \& R. Maity, 45223- (paratype, ARUN!)

Note:- Sauromatum arunachalense also shows similarity with S. gaoligongense J.C. Wang \& H. Li (1999: 61) in appearance of leaflets, outer surface of spathe tube color and long interstice but can be distinguished from both the allied species by the distinct characters discussed in Table 1. 
Table 1. Diagnostic morphological differences between Sauromatum arunachalense and its allied species.

\begin{tabular}{|c|c|c|c|}
\hline Character & $\begin{array}{l}\text { S. arunachalense } \\
\text { U.L.Tiwari, R. Maity \& } \\
\text { S.S. Dash }\end{array}$ & $\begin{array}{l}\text { S. gaoligongense J.C. } \\
\text { Wang \& H. Li }\end{array}$ & S. horsfieldii Miq. \\
\hline Leaf & $\begin{array}{l}\text { Pedatisect; } 5-7 \text { leaflets, } \\
\text { oblanceolate. }\end{array}$ & $\begin{array}{l}\text { Pedatisect; 5-13 leaflets, } \\
\text { elliptic-oblanceolate. }\end{array}$ & $\begin{array}{l}\text { Pedatisect; 7-12 } \\
\text { leaflets, elliptic-lanceolate. }\end{array}$ \\
\hline Inflorescence & Appear along with leaf. & Appear without leaf. & Appear along with leaf. \\
\hline Spathe tube & $\begin{array}{l}\text { Convolute, outside } \\
\text { longitudinally ridged when } \\
\text { mature, pinkish straw-colored } \\
\text { with purple spots throughout- } \\
\text { dark purple, inside smooth, } \\
\text { spongy-soft, greenish-white } \\
\text { towards base. }\end{array}$ & $\begin{array}{l}\text { Connate, outside purplish } \\
\text { brown with darker spots, } \\
\text { inside whitish, pale purple } \\
\text { upwards. }\end{array}$ & $\begin{array}{l}\text { Convolute, pale green } \\
\text { outside, white inside. }\end{array}$ \\
\hline Male flowers & $\begin{array}{l}2 \text { types, cream yellow, upper } \\
\text { ones comparatively long } \\
\text { stalked than lower ones, lower } \\
\text { ones minutely stalked. }\end{array}$ & $\begin{array}{l}1 \text { type, purple, minutely } \\
\text { stalked, stalk of equal length. }\end{array}$ & $\begin{array}{l}1 \text { type, cream yellow, } \\
\text { all with minute stalk } \\
\text { of equal length. }\end{array}$ \\
\hline Interstice & Long, narrow conical. & Long, cylindrical. & Short, wide conical. \\
\hline Fruit & 1-seeded. & 2-seeded. & 2-seeded. \\
\hline
\end{tabular}

Key to the species of Sauromatum

1a. Staminodes of one type

1b. Staminodes of more than one type

2a. Inflorescence appearing before leaves

S. venosum

2b. Inflorescence appearing along with leaf

3a. Leaves pubescent

3b. Leaves glabrous

4a. Spathe densely hirsute outside

S. hirsutum

4b. Spathe glabrous outside

...S. breviopilosum

5a. Spathe tube connate

5 b. Spathe tube convolute

6a. Neuter flowers more than 10, clavate, bent downwadrs; interstice between neuters and staminate flowers $c .1 \mathrm{~cm}$

...S. meghalayense

6b. Neuter flowers few, not more than 10 , spatulate, bent upwards; interstice between neuters and staminate flowers $c .2-3 \mathrm{~cm}$

S. diversifolium

7a. Leaves simple

S. giganteum

7b. Leaves pedatisect

8a. Upper staminodes spindle shaped

8b. Upper staminode subulate

9a. Spathe tube convolute 
9b. Spathe tube connate

10a. Staminal flowers of 1 type, fruit 2 -seeded

10b. Staminal flowers of 2 types, fruit 1-seeded
...S. gaoligongense

...S. horsfieldii

...S. arunachalense

\section{ACKNOWLEDGEMENTS}

The authors express their sincere gratitude to Dr. A.A. Mao, Director, Botanical Survey of India, Kolkata for facilities and encouragements and also thankful to Department of Environment and Forest of Arunachal Pradesh, Govt. of Arunachal Pradesh. RM would like to acknowledge being supported by the grants (project number GBPNI/NMHS-2017-18/LG-03/570 dt. 26/ 02/2018) under National Mission on Himalayan Studies Scheme by Ministry of Environment, Forest and Climate Change, Government of India, New Delhi.

\section{REFERENCES}

BOYCE, P.C., SOOKCHALOEM, D., HETTERSCHEID, W.L.A., GUSMAN, G., JACOBSEN, N., IDEI, T. \& V.D. NGUYEN 2012. Flora of Thailand, vol. 11(2). The Forest Herbarium, National Park, Wildlife and Plant Conservation Department, Bangkok. 101-325.

CUSIMANO N, BARRETT, M.D., HETTERSCHEID, W.L.A, \& S. RENNER 2010. A phylogeny of the Areae (Araceae) implies that Typhonium, Sauromatum, and the Australian species of Typhonium are distinct clades. Taxon 59(2): 439-447.

HENG, L. \& W.L.A. HETTERSCHEID 2010. Sauromatum Schott. In: Wu, Z.Y., Raven, P.H. \& D.Y. Hong (Eds) Flora of China, vol. 23. Science Press, Beijing, \& Missouri Botanical Garden Press, St. Louis. 36-39.

HETTERSCHEID, W.L.A. \& P.C. BOYCE 2000. A reclassification of Sauromatum Schott and new species of Typhonium Schott (Araceae). Aroideana 23: 48-55.

HETTERSCHEID, W.L.A., SOOKCHALOEM, D. \& J. MURATA 2001. Typhonium (Araceae) of Thailand: New Species and a Revised Key. Aroideana 24: 30-55.

HOOKER, J.D. 1893. Aroideae. Flora of British India, vol. 6. L. Reeve and Co., London. 490-556.
KUNTH, K.S. 1841. Sauromatum Schott In: Enum. Pl. [Kunth], vol. 3. J. G. Cotta, Stuttgart and Tübingen, Germany. 28-29.

MIQUEL, F.A.W. 1856. Flora van Nederlandsch Indie, vol. 3. Amsterdam: C.G. van der Post. 196-197.

NANGKAR, A. \& H. TAG 2018. Sauromatum nangkarense (Araceae: Areae) - A new species from Arunachal Himalaya, India. Pleione. 12(1): 227-232.

NANGKAR, A. \& H. TAG 2019. Sauromatum venosum (Dryand. ex Aiton) Kunth (Araceae : Areae): an addition to the Flora of Arunachal Pradesh in Eastern Himalaya, India. Pleione 13(1): 192-197.

ODYUO, N., ROY, D.K., DEY, S. \& A.A. MAO 2015. Sauromatum horsfieldii (Araceae: Areae): an addition to the Flora of India. Telopea 18: 227-232.

POWO 2021. Plants of the World Online. Facilitated by the Royal Botanic Gardens, Kew. Published on the Internet: https://www. plantsoftheworldonline.org/ (Accessed 30 April 2021).

SASIKALA, K., VAJRAVELU, E. \& P. DANIEL 2019. Fascicle 29: Aracea. In: Nair, V.J. \& P. Singh (Eds) Fascicles of Flora of India. Botanical Survey of India, Kolkata. 289-299.

ROY, D.K. 2018. Note on the correct identity of Sauromatum nangkarense (Araceae). Nelumbo 60(2): 162.

SCHOTT, H.W. 1832. Araceae. In: SCHOTT, H.W. \& S. ENDLICHER (Eds) Meletemata botanica. Gerold, Vienna. 16-22.

TALUKDAR, A.D., ROY, D.K., SINHA, B.K., M. DUTTA CHOUDHURY 2014. Sauromatum meghalayense (Araceae; Tribe: Areae), a new species from Meghalaya, India. NeBIO 5(3): 1-3.

WANG, Z.-L. \& H. LI, 1999. Sauromatum gaoligongense (Araceae), a new species from Gaoligong mountains. Acta Bot. Yunnan., suppl. 11:61-64. 